\title{
SENTINEL-1 IMAGE CLASSIFICATION FOR CITY EXTRACTION BASED ON THE SUPPORT VECTOR MACHINE AND RANDOM FOREST ALGORITHMS
}

\author{
Ali Jamali ${ }^{1 *}$ and Alias Abdul Rahman ${ }^{2}$ \\ ${ }^{1}$ Faculty of Surveying Engineering, Apadana Institute of Higher Education, Shiraz, Iran- ali.jamali.65@ gmail.com \\ ${ }^{2}$ Universiti Teknologi Malaysia (UTM), Faculty of Geoinformation and Real Estate- alias@utm.my
}

KEYWORDS: SAR, Support Vector Machine, Random Forest, LULCC, R statistical packages

\begin{abstract}
:
Environmental change monitoring in earth sciences needs land use land cover change (LULCC) modeling to investigate the impact of climate change phenomena such as droughts and floods on earth surface land cover. As land cover has a direct impact on Land Surface Temperature (LST), the Land cover mapping is an essential part of climate change modeling. In this paper, for land use land cover mapping (LULCM), image classification of Sentinel-1A Synthetic Aperture Radar (SAR) Ground Range Detected (GRD) data using two machine learning algorithms including Support Vector Machine (SVM) and Random Forest (RF) are implemented in R programming language and compared in terms of overall accuracy for image classification. Considering eight different scenarios defined in this research, RF and SVM classification methods show their best performance with overall accuracies of 90.81 and 92.09 percent respectively.
\end{abstract}

\section{INTRODUCTION}

Land cover is a fundamental factor that links and effect with many parts of the human and physical environment (Foody, 2002). The change in land cover is considered as an important factor of global change affecting ecological systems (Vitousek, 1994) with an impact on the earth that is linked with climatic change (Skole, 1994). Land cover mapping (Grippa et al., 2018) and monitoring are one of the key applications of earth observation satellites sensor data which is an important factor to asses results of climate change in the recent years.

On the other hand, changes in land cover affect the climate through changes in the composition of greenhouse gasses such as carbon dioxide (Betts et al., 2007; Bonan, 2008; Bala et al., 2007). Up to date, land use land cover (LULC) statistics are a need for policy and decision making which has an effect on economy and society (Costa et al., 2018).

According to Rodriguez-Galiano et al. (2012), there are several issues for large area land cover monitoring including:

1. First, complex landscapes are difficult to monitor due to sudden changes in environmental gradients (e.g. moisture, elevation, and temperature) and a legacy of past interference (Rogan and Miller, 2006). Such heterogeneous landscapes are defined by land-cover categories that are complicated to be defined spectrally due to low inter-class separability and high intra-class variability.

2. Second, there is a need for algorithms that can be interpreted readily and automated as well as to be easily run with user-defined parameters that are simple to adjust.
3. Third, a promising land-cover classification algorithm for large area mapping relies on the capability of the algorithm to work with noisy observations, a complex measurement space, and a few numbers of training data compared to the size of the study area (DeFries and Chan, 2000; Rogan et al., 2008).

A wide range of classification methods has been utilized to map land cover using remotely sensed data. Classification methods vary from unsupervised algorithms such as K-means clustering to parametric supervised algorithms such as maximum likelihood (Otukei and Blaschke, 2010); to machine learning algorithms such as artificial neural networks (Duro et al, 2012), SVMs (Mountrakis et al., 2011), decision trees (Breiman, 1984; Hua et al., 2017), and ensembles of classifiers (Breiman, 1996).

The usual purpose of land cover classification is to produce a thematic map of the land cover. Land cover is the material at the ground, such as vegetation, water, soil, and man-made structures. (Fisher and Unwin, 2005). The number and kind of land cover classes in the image that can be defined vary significantly depending on the sensor resolutions.

For land monitoring of forests, water, soil, agriculture, emergency mapping support for natural disasters including flooding, landslide, earthquakes Sentinel-1A/1B satellites were launched. Sentinel-1 satellites carry a C-SAR sensor, which has medium and high-resolution imaging in allweather conditions. The C-SAR can obtain night imagery and detecting small movement on the ground, which makes it useful for land and sea monitoring. 
SAR is known for its day-and-night and all-weather imaging ability. In last two decades, for SAR image interpretation, several methods including statistical-based (Tison et al., 2004; Marques et al., 2012), texture-based (Haralick and Shanmugam, 1973; Torres-Torriti and Jouan, 2001) and model-based (Li, 2009; Lafferty et al., 2001) methods have introduced (He et al., 2017). A SAR signal has phase and amplitude information where the phase is the fraction of one complete sine wave cycle and the amplitude is the strength of the radar response.

The phase of the SAR image is determined by the distance between the ground targets and the satellite antenna. According to the European Space Agency (ESA), Sentinel-1 can simultaneously collect several different images from the same series of pulses by using its antenna to receive specific polarisations. Basically, Sentinel-1 is a phase-preserving dual polarisation SAR system. It can transmit a signal in both horizontal $(\mathrm{H})$ and vertical $(\mathrm{V})$ polarisation and then receive in both $\mathrm{H}$ and $\mathrm{V}$ polarisations. Dual polarisation Level-1 Single Look Complex (SLC) products contain complex values. In addition to the backscatter intensity that can be measured from every single polarisation, the inter-channel phase information allows performing enhanced analysis of backscattering properties.

Random Forest algorithm has been highly utilized for image classification (Jozdani et al., 2019; Gapper et al., 2019; Traoré et al., 2019). The Support Vector Machine algorithm can achieve high classification precisions with small training collections (Foody and Mathur, 2006). Additionally, the SVM method is a robust approach for low noise levels with some mislabeled training information (Pelletier et al., 2017). However, some researchers outperformed the RF and ANN algorithms in their researches (Pelletier et al., 2017; Karantzalos et al., 2015).

In this research, for Sentinel-1A image classification for city extraction (i.e. build-up regions), Random Forest (RF) and Support Vector Machine (SVM) algorithms are implemented in the $\mathrm{R}$ programming language and compared in term of overall accuracy for image classification. Following this Section, in Section 2, classification methods are discussed. Study area and data used in this research are discussed in Section 3. Results of image classification based on the RF and SVM are presented in Section 4. Discussions and conclusions are presented in Section 5.

\section{METHODS}

Two machine learning classification methods including RF and SVM in $\mathrm{R}$ programming language are researched. Dealing with large and complex datasets, machine learning algorithms are more accurate and efficient compared to conventional parametric algorithms, (Rodriguez-Galiano et al., 2012). Both RF and SVM are state-of-the-art machine learning algorithms for image classification.
RF (Breiman, 2001) method is an extension of classification and regression trees (CART; Breiman et al., 1984). RF method is an ensemble learning technique which is increasingly used in land-cover classification using multispectral and hyperspectral satellite sensor imagery. RF creates several trees based on random bootstrapped of the training dataset samples. RF runs random binary trees that creates a subset of the training over bootstrapping method, from the initial dataset, a random selection of the training data is selected and implemented to construct the model, out of bag (OOB) is the data which is not included (Catani et al. 2013). The number of trees (ntree), and the number of variables (mtry) are two parameters which are needed to be tuned in an RF method.

SVMs (Vapnik, 1998) uses a simple linear method to the data but in a high-dimensional feature space non-linearly related to the input space, but in practice, it does not use any computations in that high-dimensional space. The combined the state-of-the-art performance and simplicity on many learning problems (regression and classification) has increased the popularity of the SVMs (Leo et al., 2006). SVM is a supervised machine learning technique that is implemented based on the Structural Risk Minimization (SRM) principle and statistical learning theory (Tehrany et al. 2015). SVMs have higher accuracies compared with the traditional approaches but the results rely on the kernel used, choice of parameters for the chosen kernel and the method used to generated SVM (Huang et al., 2002).

\section{STUDY AREA AND DATA USED}

The data is from Sentinel-1A GRD data belonging to 15th November 2018 data set of Shiraz city in WGS 84 / UTM zone $39 \mathrm{~N}$ (Figure 1). Shiraz is located in the south of Iran which is built in a green plain at the foot of the Zagros Mountains, 1,500 meters (4,900 feet) above sea level. For Sentinel-1A image classification, eight scenarios with different polarization data combination are used (see Table $1)$.

For this study, Sentinel-1A GRDH data is selected due to its high resolutions of $88 \times 87$ meters and the fact that these data are free of charge which make them a valuable data source for researchers and academia working on effects of climate change phenomena on LULCC. Zhou et al. (2016) proposed a state of the art for image classification method of polarimetric SAR image classification using deep convolutional neural networks and its advantages over conventional image classification methods where in this research two state of the art machine learning algorithms (i.e. RF and SVM) are used and compared in terms of overall accuracy. 
The International Archives of the Photogrammetry, Remote Sensing and Spatial Information Sciences, Volume XLII-4/W16, 2019 6th International Conference on Geomatics and Geospatial Technology (GGT 2019), 1-3 October 2019, Kuala Lumpur, Malaysia

Landsat True Color Composite

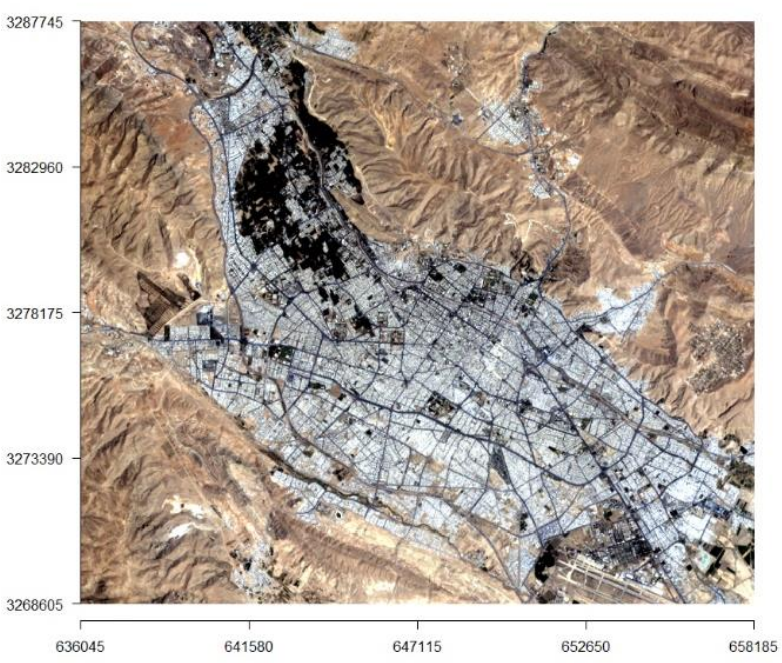

Landsat False Color Composite

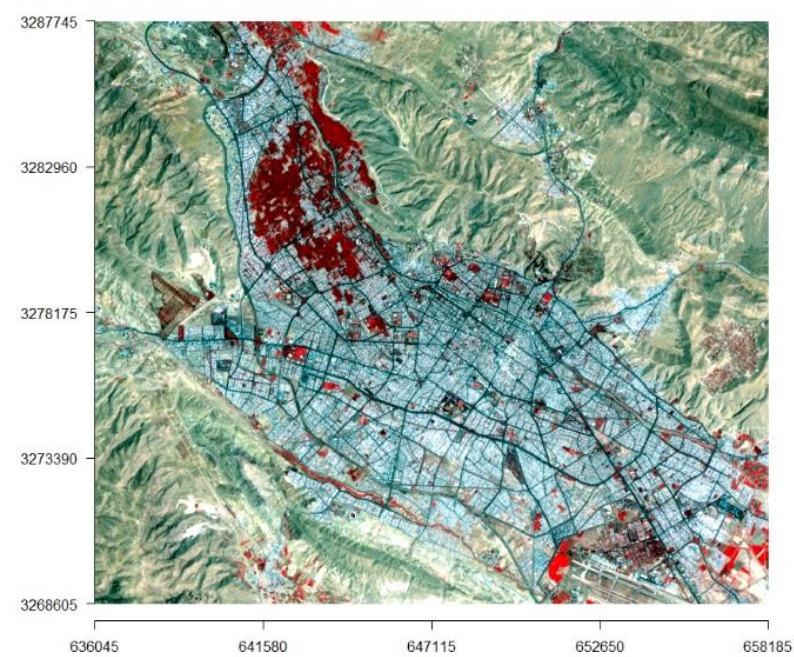

Figure 1. Study area.

\section{RESULTS}

\subsection{Pre-processing}

Sentinel-1A/1B SAR images are required to be pre-processed before any image classification. In this research, using Sentinel Application Platform (SNAP) software, for image pre-processing a graph is created in GraphBuilder as follows (see Figure 2):

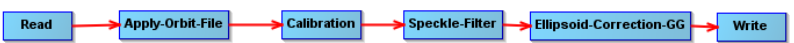

Figure 2. The implemented pre-processing graph of Sentinel$1 \mathrm{~A}$ in SNAP.

In the first step, the orbit state vector is redefined with a polynomial degree of three as metadata of a SAR product are generally not accurate and can be corrected with the precise orbit files which are available days-to-weeks after the generation of the product. In the second step, produces level1 images are required to be radiometrically corrected because they do not include radiometric corrections and significant radiometric bias remains. The radiometric correction is necessary for the pixel values to truly represent the radar backscatter of the reflecting surface.

In the third step, speckles which are caused by random constructive and destructive interference of the de-phased but coherent return waves scattered by the elementary scatters within each resolution cell are filtered with Lee Sigma with a window size of $7 \mathrm{by} 7$, sigma of 0.9 and target window size of 3by3. In the final step, for ellipsoidal correction of Sentinel1A SAR image, Geo-location Grid is used (see Figure 3).

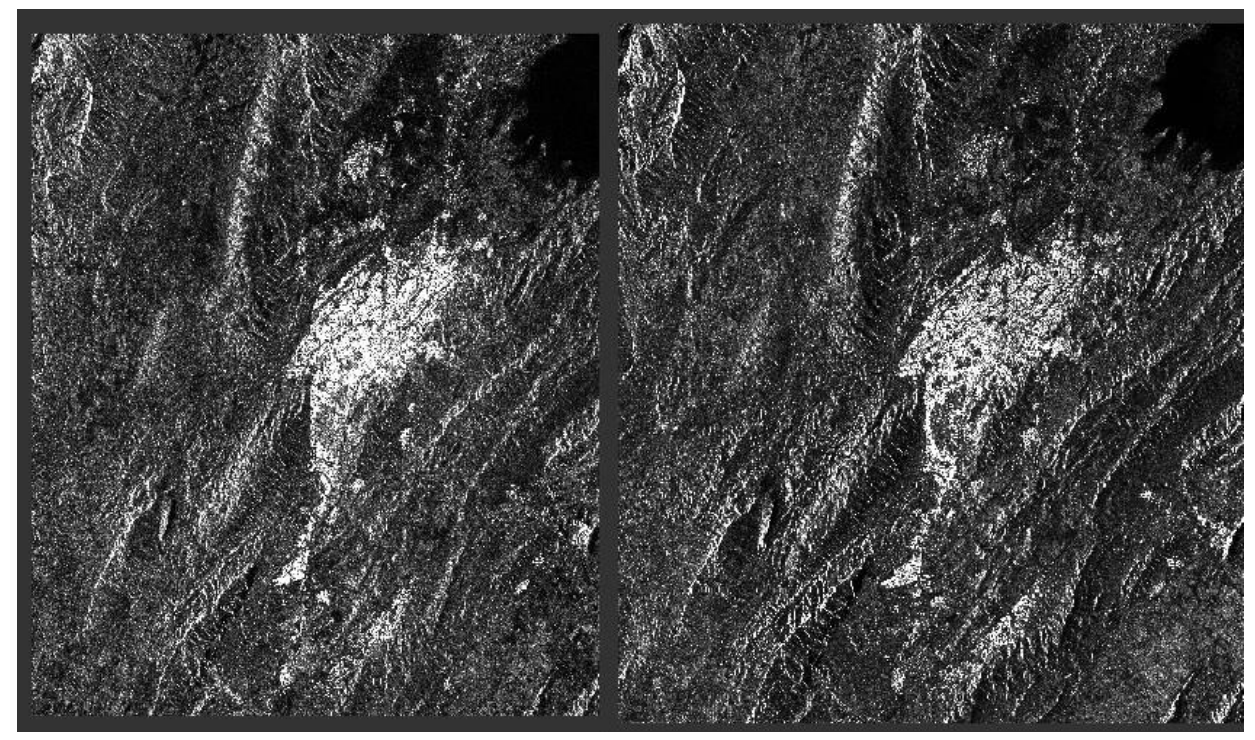


The International Archives of the Photogrammetry, Remote Sensing and Spatial Information Sciences, Volume XLII-4/W16, 2019 6th International Conference on Geomatics and Geospatial Technology (GGT 2019), 1-3 October 2019, Kuala Lumpur, Malaysia

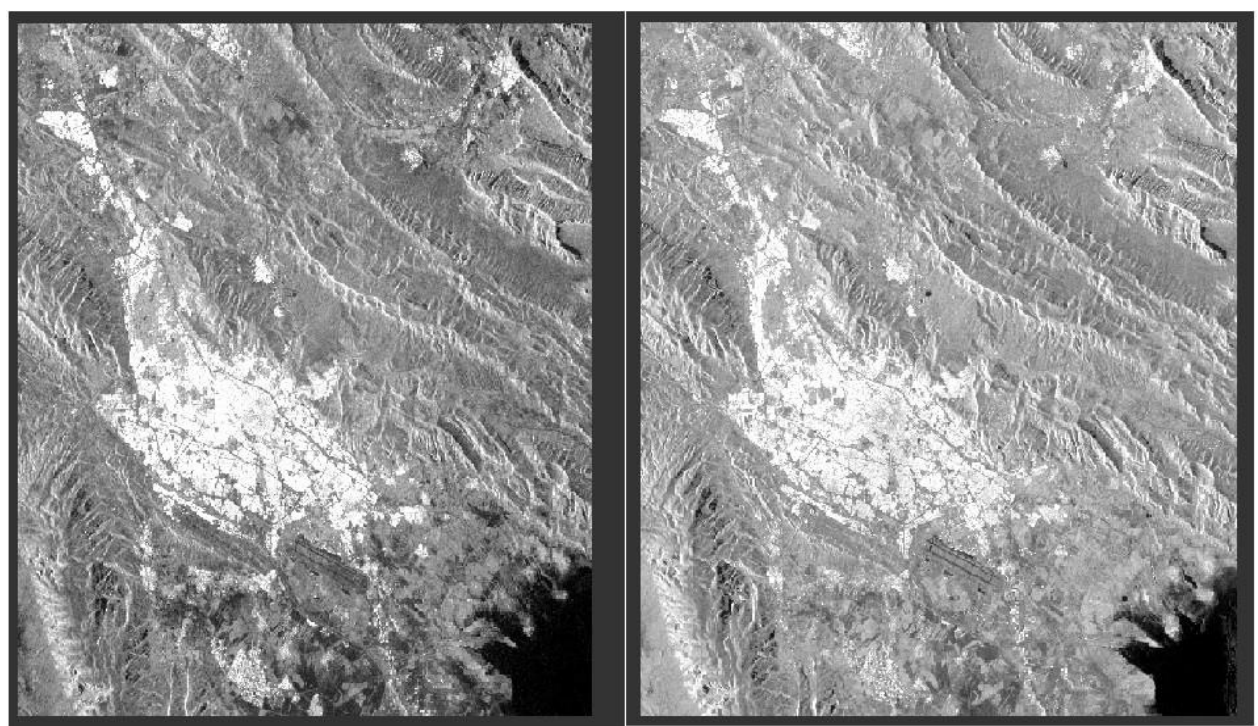

Figure 3. a) Intensity_VH polarization data (top left) b) Intensity_VV polarization data (top right) c) Saigma_0_VH_db polarization data (down left) d) Saigma_0_VV_db polarization data (down right)

\section{2 image classification}

Most of the classification methods take a formula such as $\mathrm{Y}=\mathrm{X} \_1+\mathrm{X} \_2$, to find dependent and independent variables where $\mathrm{Y}$ is a function of $\mathrm{X} 1$ and $\mathrm{X} 2$.

Considering VV and VH polarimetry data (see Figure 4) plus dual polarization ratio, dual polarization difference and dual polarization multiple for Sentinel-1A GRD data, in $\mathrm{R}$ programming language, the classification formula is written by (see Equation 1):

classes $\sim(\mathrm{VV})+(\mathrm{VH})+(\mathrm{VV} / \mathrm{VH})+(\mathrm{VV}+\mathrm{VH})+(\mathrm{VV}-\mathrm{VH})+$ $(\mathrm{NDDPI})+(\mathrm{abs}(\mathrm{VV} * \mathrm{VH}))(1)$

where

Normalized Diffrence Dual Polarization Index = NDDPI $=\frac{V V-V H}{V V+V H}$

Sentinel-1A images are classified by five materials including build-up, roads, soil, water and vegetation regions to evaluate the performance of two image classification methods including RF (see Figure 5) and SVM (see Figure 6) algorithms.

Table 1. dataset of experiments for land cover classification

\begin{tabular}{|l|l|l|}
\hline Scenario & $\begin{array}{l}\text { Number of } \\
\text { variables }\end{array}$ & Variables \\
\hline 1 & 1 & $(\mathrm{VV}+\mathrm{VH}) / 2$ \\
\hline 2 & 2 & $\mathrm{VV}, \mathrm{VH}$ \\
\hline 3 & 3 & $\mathrm{VV}, \mathrm{VH},(\mathrm{VV}+\mathrm{VH})$ \\
\hline 4 & 3 & $\mathrm{VV}, \mathrm{VH},(\mathrm{VV}-\mathrm{VH})$ \\
\hline 5 & 3 & $\mathrm{VV}, \mathrm{VH},(\mathrm{VV} / \mathrm{VH})$ \\
\hline 6 & 3 & $\mathrm{VV}, \mathrm{VH}, \mathrm{NDDPI}$ \\
\hline 7 & 4 & $\begin{array}{l}\mathrm{VV}, \mathrm{VH},(\mathrm{VV}+\mathrm{VH}),(\mathrm{VV}- \\
\mathrm{VH})\end{array}$ \\
\hline
\end{tabular}

\begin{tabular}{|l|l|l|}
\hline 8 & 7 & $\begin{array}{l}\mathrm{VV}, \mathrm{VH},(\mathrm{VV}-\mathrm{VH}), \\
(\mathrm{VV}+\mathrm{VH}),(\mathrm{VV} / \mathrm{VH}), \\
(\mathrm{NDDPI}),(\mathrm{abs}(\mathrm{VV} * \mathrm{VH}))\end{array}$ \\
\hline
\end{tabular}

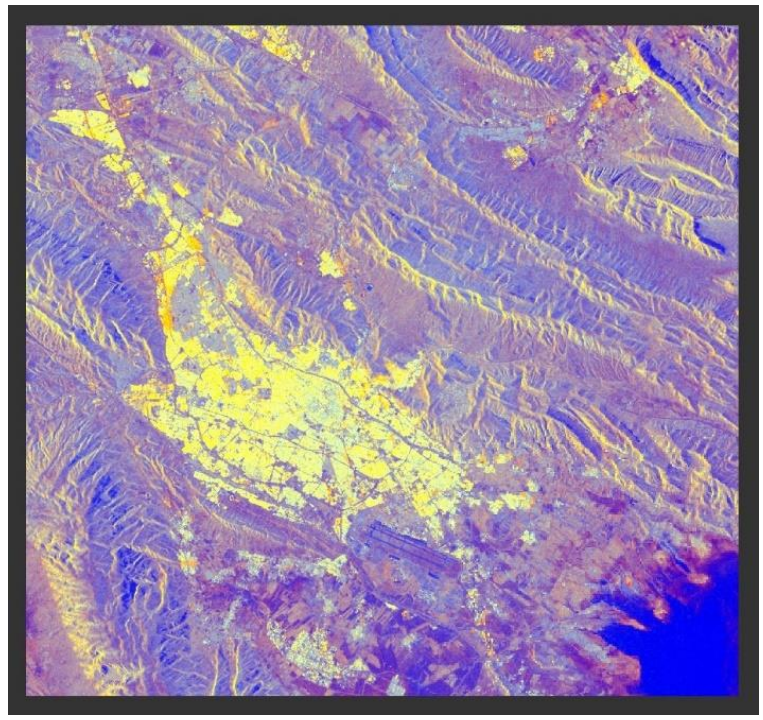

Figure 4. Dual Polarization ratio of VV+ VH of Shiraz city.

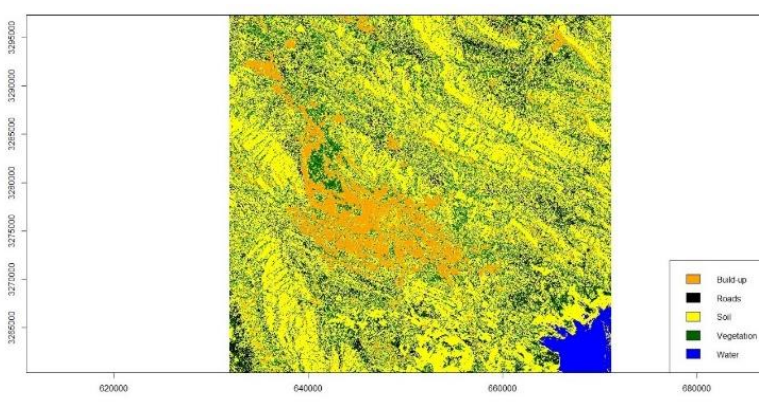

Figure 5. Image classification using Random Forest classifier within $\mathrm{R}$ programming language. 


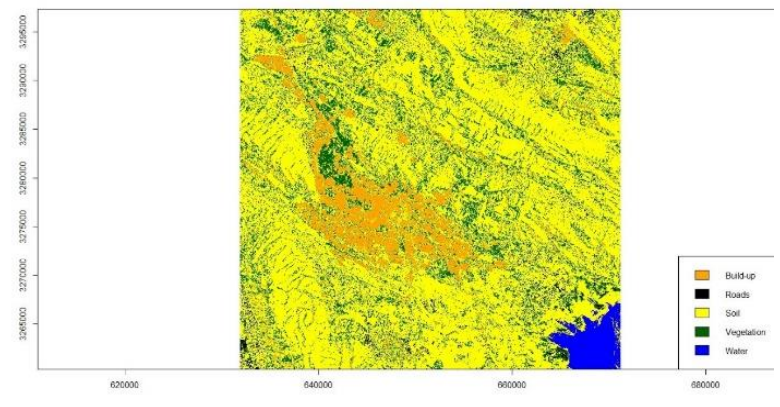

Figure 5. Image classification using Support Vector Machine classifier within $\mathrm{R}$ programming language.

Considering eight different scenarios with different defined dual polarization data information, RF algorithm has its best performance in scenario 4 with $\mathrm{VV}, \mathrm{VH},(\mathrm{VV}-\mathrm{VH})$ polarization data. On the other hand, the SVM algorithm shows its best performance in scenario 3 which has $\mathrm{VV}, \mathrm{VH}$, $(\mathrm{VV}+\mathrm{VH})$ polarization information (see Table 4).

Table 4. Overall accuracy of RF and SVM image classification methods in seven defined scenarios.

\begin{tabular}{|l|l|l|}
\hline Scenario & $\begin{array}{l}\text { RF overall } \\
\text { accuracy }\end{array}$ & $\begin{array}{l}\text { SVM overall } \\
\text { accuracy }\end{array}$ \\
\hline 1 & 88.83 & 91.76 \\
\hline 2 & 90.60 & 91.97 \\
\hline 3 & 90.74 & 92.09 \\
\hline 4 & 90.81 & 91.78 \\
\hline 5 & 90.75 & 91.97 \\
\hline 6 & 90.65 & 92.03 \\
\hline 7 & 90.63 & 91.86 \\
\hline 8 & 90.62 & 90.63 \\
\hline
\end{tabular}

\section{CONCLUSIONS}

Free and commercial Earth Observation (EO) satellites sensor data are a key factor for large area environmental monitoring. Due to several climate change phenomena (e.g. increase of temperature due to greenhouse gasses) in recent years and their impact on the land cover change and vice versa; the effect of land cover changes on earth climate, image classification for large area environments is a necessity. There are several statistical and mathematical image classification algorithms where in this research performance of two machine learning algorithms for a dataset of Shiraz city in Iran in eight different scenarios are researched. To extract city areas, SVM algorithm has better overall accuracy for Sentinel-1A GRD data image classification over RF algorithm for the dataset used in this research.

\section{REFERENCES}

Bala, G., Caldeira, K., Wickett, M., Phillips, T., Lobell, D., Delire, C., Mirin, A., (2007). Combined climate and carboncycle effects of large-scale deforestation. Proceedings of the National Academy of Sciences 104 (16), 6550-6555.
Betts, R., Falloon, P., Goldewijk, K., Ramankutty, N., (2007). Biogeophysical effects of land use on climate: model simulations of radiative forcing and large-scale temperature change. Agricultural and Forest Meteorology 142 (2-4), 216233.

Bonan, G., (2008). Forests and climate change: forcings, feedbacks, and the climate benefits of forests. Science 320 (5882), 1444-1449.

Breiman, L., (1984). Classification and Regression Trees. Chapman \& Hall/CRC.

Breiman, L., (1996). Bagging predictors. Machine Learning 24 (2), 123-140.

Breiman, L., (2001). Random forests. Machine learning, 45(1), 5-32.

Catani F, Lagomarsino D, Segoni S, Tofani V., (2013). Landslide susceptibility estimation by random forests technique: sensitivity and scaling issues. Nat Hazards Earth Syst Sci 13:2815-2831.

Costa, H., Almeida, D., Vala, F., Marcelino, F., \& Caetano, M. (2018). Land cover mapping from remotely sensed and auxiliary data for harmonized official statistics. ISPRS International Journal of Geo-Information, 7(4), 157.

DeFries, R.S., Chan, J.C.-W., (2000). Multiple criteria for evaluating machine learning algorithms for land cover classification from satellite data. Remote Sensing of Environment 74 (3), 503-515.

Duro, D. C., Franklin, S. E., \& Dubé, M. G., (2012). A comparison of pixel-based and object-based image analysis with selected machine learning algorithms for the classification of agricultural landscapes using SPOT-5 HRG imagery. Remote Sensing of Environment, 118, 259-272.

Foody, G.M. and A. Mathur, The use of small training sets containing mixed pixels for accurate hard image classification: Training on mixed spectral responses for classification by a SVM. Remote Sens. Environ, 2006. 103: p. 179-189.

Erdas Inc., (1999). Erdas Field Guide. Erdas Inc., Atlanta, Georgia.

Fisher, P. F. and Unwin, D. J., eds. (2005). Representing GIS. Chichester, England: John Wiley \& Sons.

Foody, G.M., (2002). Status of land cover classification accuracy assessment. Remote Sensing of the Environment 80, 185-201.

Gapper, J. J., El-Askary, H., Linstead, E., \& Piechota, T. (2019). Coral Reef Change Detection in Remote Pacific Islands Using Support Vector Machine Classifiers. Remote Sensing, 11(13), 1525.

Grippa, T., Georganos, S., Zarougui, S., Bognounou, P., Diboulo, E., Forget, Y., ... \& Wolff, E., (2018). Mapping Urban Land Use at Street Block Level Using OpenStreetMap, Remote Sensing Data, and Spatial Metrics. ISPRS International Journal of GeoInformation, 7(7), 246. 
Han, S., Ren, F., Wu, C., Chen, Y., Du, Q., \& Ye, X., (2018). Using the TensorFlow Deep Neural Network to Classify Mainland China Visitor Behaviours in Hong Kong from Check-in Data. ISPRS International Journal of GeoInformation, 7(4), 158.

Haralick, R. M., \& Shanmugam, K. (1973). Textural features for image classification. IEEE Transactions on systems, man, and cybernetics, (6), 610-621.

He, C., Liu, X., Kang, C., Chen, D., \& Liao, M. (2017). Attribute Learning for SAR Image Classification. ISPRS International Journal of Geo-Information, 6(4), 111.

Hua, L., Zhang, X., Chen, X., Yin, K., \& Tang, L., (2017). A Feature-Based Approach of Decision Tree Classification to Map Time Series Urban Land Use and Land Cover with Landsat $5 \mathrm{TM}$ and Landsat $8 \mathrm{OLI}$ in a Coastal City, China. ISPRS International Journal of GeoInformation, 6(11), 331.

Huang, C., Davis, L.S., Townshed, J.R.G., (2002). An assessment of support Vector Machines for Land cover classification. International Journal of Remote sensing 23, 725-749.

JARS, (1993). Remote Sensing Note. Japan Association on Remote Sensing. Available at http://www.jars1974.net/pdf/rsnote_e.html.

Jozdani, S. E., Johnson, B. A., \& Chen, D. (2019). Comparing Deep Neural Networks, Ensemble Classifiers, and Support Vector Machine Algorithms for Object-Based Urban Land Use/Land Cover Classification. Remote Sensing, 11(14), 1713.

Karantzalos, K., D. Bliziotis, and A. Karmas, A scalab legeo spatial web service for near real-time, high-resolution land cover mapping. IEEE J. Sel. Top. Appl. Earth Obs. Remote Sens, 2015. 8: p. 4665-4674.

Lafferty, J., McCallum, A., \& Pereira, F. C. (2001). Conditional random fields: Probabilistic models for segmenting and labeling sequence data.

Li, S. Z. (2009). Markov random field modeling in image analysis. Springer Science \& Business Media.

Leo, B., Friedman, J. H., Olshen, R. A., \& Stone, C. J., (1984). Classification and regression trees. Wadsworth International Group.

Marques, R. C. P., Medeiros, F. N., \& Nobre, J. S. (2012). SAR Image Segmentation Based on Level Set Approach and $\{$ \cal $\mathrm{G}\} \mathrm{A}^{\wedge} 0$ Model. IEEE transactions on pattern analysis and machine intelligence, 34(10), 2046-2057.

Mountrakis, G., Im, J., Ogole, C., (2011). Support vector machines in remote sensing: a review. ISPRS Journal of Photogrammetry and Remote Sensing 66 (3), 247-259.

NASA, (2013). Landsat 7 Science Data User's Handbook. Available at http://landsathandbook.gsfc.nasa.gov

Otukei, J. R., \& Blaschke, T., (2010). Land cover change assessment using decision trees, support vector machines and maximum likelihood classification algorithms. International Journal of Applied Earth Observation and Geoinformation, 12, S27-S31.

Pelletier, C., et al., Effect of Training Class Label Noise on Classification Performances for Land Cover Mapping with Satellite Image Time Series. Remote Sens, 2017. 9: p. 173.

Rodriguez-Galiano, V. F., Ghimire, B., Rogan, J., ChicaOlmo, M., \& Rigol-Sanchez, J. P., (2012). An assessment of the effectiveness of a random forest classifier for land-cover classification. ISPRS Journal of Photogrammetry and Remote Sensing, 67, 93-104.

Rogan, J., Miller, J., (2006). Integrating GIS and remotely sensed data for mapping forest disturbance and change. In: Franklin, M.W.A.S. (Ed.), Understanding Forest Disturbance and Spatial Pattern: Remote Sensing and GIS Approaches. CRC Press, Boca Raton, FL, pp. 133-172.

Rogan, J., Franklin, J., Stow, D., Miller, J., Woodcock, C., Roberts, D., (2008). Mapping land-cover modifications over large areas: a comparison of machine learning algorithms. Remote Sensing of Environment 112 (5), 2272-2283.

Skole, D.L., (1994). Data on global land cover change: acquisition assessment and analysis. In: Turner, II, W.B. (Ed.), Changes in Land Use and Land Cover: A Global Perspective. Cambridge University Press, Cambridge, pp. 437-471.

Traoré, F., Bonkoungou, J., Compaoré, J., Kouadio, L., Wellens, J., Hallot, E., \& Tychon, B. (2019). Using MultiTemporal Landsat Images and Support Vector Machine to Assess the Changes in Agricultural Irrigated Areas in the Mogtedo Region, Burkina Faso. Remote Sensing, 11(12), 1442 .

Tehrany MS, Pradhan B, Mansor S, Ahmad N., (2015). Flood susceptibility assessment using GIS-based support vector machine model with different kernel types. Catena 125:91101. doi:10.1016/j.catena.2014.10.017.

Tison, C., Nicolas, J. M., Tupin, F., \& Maître, H. (2004). A new statistical model for Markovian classification of urban areas in high-resolution SAR images. IEEE transactions on geoscience and remote sensing, 42(10), 2046-2057.

Torres-Torriti, M., \& Jouan, A. (2001). Gabor vs. GMRF features for SAR imagery classification. In Image Processing, 2001. Proceedings. 2001 International Conference on (Vol. 3, pp. 1043-1046). IEEE.

Vapnik, V. (1998). Statistical Learning Theory. John Wiley\&Sons. Inc., New York.

Vitousek, P.M., (1994). Beyond global warming: ecology and global change. Ecology 75, 1861-1876.

Zhou, Y., Wang, H., Xu, F., \& Jin, Y. Q. (2016). Polarimetric SAR image classification using deep convolutional neural networks. IEEE Geoscience and Remote Sensing Letters, 13(12), 1935-1939.

Revised August 2019 